\title{
Exact localized eigenstates for an extended Bose-Hubbard model with pair-correlated hopping
}

Peter Jason and Magnus Johansson

\section{Linköping University Post Print}

N.B.: When citing this work, cite the original article.

Original Publication:

Peter Jason and Magnus Johansson, Exact localized eigenstates for an extended BoseHubbard model with pair-correlated hopping, 2012, Physical Review A. Atomic, Molecular, and Optical Physics, (85), 1, 016603(R).

http://dx.doi.org/10.1103/PhysRevA.85.011603

Copyright: American Physical Society http://www.aps.org/

Postprint available at: Linköping University Electronic Press

http://urn.kb.se/resolve?urn=urn:nbn:se:liu:diva-73926 


\title{
Exact localized eigenstates for an extended Bose-Hubbard model with pair-correlated hopping
}

\author{
${\text { Peter Jason* }{ }^{*} \text { and Magnus Johansson }}^{\dagger}$ \\ Department of Physics, Chemistry and Biology, Linköping University, SE-581 83 Linköping, Sweden
}

(Received 2 September 2011; published 5 January 2012)

\begin{abstract}
We show that a Bose-Hubbard model extended with pair-correlated hopping has exact eigenstates, quantum lattice compactons, with complete single-site localization. These appear at parameter values where the oneparticle tunneling is exactly canceled by nonlocal pair correlations, and correspond in a classical limit to compact solutions of an extended discrete nonlinear Schrödinger model. Classical compactons at other parameter values, as well as multisite compactons, generically get delocalized by quantum effects, but strong localization appears asymptotically for increasing particle number.
\end{abstract}

DOI: 10.1103/PhysRevA.85.011603

PACS number(s): 03.75.Lm, 05.30.Jp, 05.45.Yv, 67.85.-d

Introduction. Intrinsically localized modes ("discrete breathers") appear as generic excitations in classical nonlinear Hamiltonian lattices [1], and a basic model describing the (quasi)particle localization is the discrete nonlinear Schrödinger (DNLS) equation [1,2]. For applications, seeking an optimal localization may be important. In this respect, compactons, first discovered as classical waves with compact support in presence of nonlinear dispersion [3], are interesting as their tail-less nature prohibits mutual interactions unless they are in direct contact. In generic classical oscillator lattices discreteness destroys the compactness into superexponential decay [1], but for certain models the amplitude at a given site may be tuned to exactly vanish the coupling to its neighbor, yielding exact "discrete compactons" [4]. In particular, compactons localized on one or several sites were found, under certain parameter restrictions, for an extended DNLS model of an optical waveguide array with nonlinear coupling [5]. It was recently suggested [6] that stable compactons analogous to those of Ref. [5] could be experimentally realized in the mean-field regime of a BEC in an optical lattice, using a rapidly time-varying magnetic field to effectively create nonlinear dispersion.

However, to our knowledge, the question as to whether discrete compactons in (semi)classical (mean-field) models can be continued into strictly localized exact solutions of a corresponding quantum lattice model was not addressed up to now. A basic quantum lattice model, describing a variety of phenomena for interacting bosonic (quasi)particles in deep periodic potentials, is the Bose-Hubbard (BH) model. It is a standard tool for analyzing the many-body physics of strongly correlated cold atoms in optical lattices $[7,8]$, where predictions such as superfluid-insulator transition of the ground state $[9,10]$ were experimentally realized [11]. The same model was introduced to describe local-mode molecular vibrations [12,13] and "quantum discrete breathers" [14], which are quantum counterparts to classical intrinsically localized modes. When the number of particles per lattice site becomes large, a mean-field approach treating the Bose condensate as a classical field can be employed [15], leading to a lattice Gross-Pitaevskii equation equivalent to the DNLS

\footnotetext{
*petej@ifm.liu.se

${ }^{\dagger}$ mjn@ifm.liu.se; https://people.ifm.liu.se/majoh
}

equation [16]. Several effects predicted from the classical DNLS theory were also experimentally observed with cold atoms $[1,15]$.

The BH model essentially describes the competition between one-particle tunneling and on-site interactions. However, in some situations more complicated processes must be taken into account. In particular, atom-atom interactions are finite ranged, leading to pair correlations in the tunneling between neighboring lattice sites which have been shown to be relevant in various setups and described by an extended Bose-Hubbard (EBH) model in several theoretical [17-21] as well as experimental [22] works. Taking the mean-field limit of this EBH model turns the pair tunneling into nonlinear coupling terms, and the semiclassical dynamics is described by an extended DNLS equation $[23,24]$ equivalent to that of Ref. [5].

In this Rapid Communication we prove that the classical one-site compactons of Ref. [5], under an additional parameter restriction, correspond to exact eigenstates of the $\mathrm{EBH}$ model with complete single-site localization, quantum lattice compactons (QLCs). We also show that exact localization is generically destroyed by quantum fluctuations for classical multisite compactons, as well as for one-site compactons not fulfilling the additional parameter condition, and demonstrate this effect numerically. A two-site compactonlike eigenstate, consisting mainly of two-site basis states with Gaussian distribution, is shown to appear asymptotically for increasing particle number.

Model. We consider the EBH Hamiltonian [17-22]:

$$
\begin{aligned}
\hat{H}_{\mathrm{EBH}}= & \frac{1}{2} \sum_{i}\left\{Q_{1} \hat{N}_{i}+Q_{2} \hat{a}_{i+1}^{\dagger} \hat{a}_{i}+Q_{3} \hat{N}_{i}^{2}\right. \\
& +2 Q_{4}\left[2 \hat{N}_{i} \hat{N}_{i+1}+\left(\hat{a}_{i+1}^{\dagger}\right)^{2}\left(\hat{a}_{i}\right)^{2}\right] \\
& \left.+4 Q_{5}\left[\left(\hat{a}_{i}^{\dagger}\right)^{2}+\left(\hat{a}_{i+1}^{\dagger}\right)^{2}\right] \hat{a}_{i} \hat{a}_{i+1}\right\}+ \text { H.c. }
\end{aligned}
$$

Here $\hat{a}_{i}^{\dagger}\left(\hat{a}_{i}\right)$ is the bosonic creation (annihilation) operator and $\hat{N}_{i}=\hat{a}_{i}^{\dagger} \hat{a}_{i}$ the corresponding number operator for particles at site $i$ (H.c. is the Hermitian conjugate). With a notation analogous to Ref. [5], the model contains five $Q$ parameters connected to different processes; e.g., $\hat{a}_{i+1}^{\dagger} \hat{a}_{i}$ and $\left(\hat{a}_{i+1}^{\dagger}\right)^{2}\left(\hat{a}_{i}\right)^{2}$ can be interpreted as one particle and a particle pair, respectively, tunneling from site $i$ to $i+1$, and $\left(\hat{a}_{i+1}^{\dagger}\right)^{2} \hat{a}_{i} \hat{a}_{i+1}$ as tunneling of one particle from site $i$ to $i+1$, but only if 
there already resides at least one other particle at $i+1$. The other terms can be interpreted in similar fashions [18]. Putting $Q_{4}$ and $Q_{5}$ to zero retrieves the original $\mathrm{BH}$ model. Since $\hat{N} \equiv \sum_{i} \hat{N}_{i}$ commutes with $\hat{H}_{\text {EBH }}$, the $Q_{1}$ term can be removed by a shift of the zero energy. One more parameter can be fixed by rescaling the energy and the other parameters, reducing the number of independent $Q$ parameters to three [25]. Here we set $Q_{3}=1$, leaving $Q_{2}, Q_{4}$, and $Q_{5}$ as independent variables [26]. Thus, our discussion will be in the context of a repulsive on-site interaction, but the attractive case $\left(Q_{3}=-1\right)$ is also covered via the simple transformation $\hat{a}_{i}^{(\dagger)} \rightarrow(-1)^{i} \hat{a}_{i}^{(\dagger)}, Q_{4} \rightarrow-Q_{4}$, $\hat{H}_{\text {EBH }} \rightarrow-\hat{H}_{\text {EBH }}$.

To compare with the classical model [5], a finite limit of (1) for $N \equiv\langle\hat{N}\rangle \rightarrow \infty$ must be properly defined. For this, we consider relative rather than absolute particle numbers by redefining the annihilation (creation) operator as $\hat{a}^{\prime(\dagger)}=$ $\hat{a}^{(\dagger)} / \sqrt{N}$ and number operator $\hat{N}^{\prime}=\hat{N} / N$, and rescale the Hamiltonian as $\hat{H}_{\mathrm{EBH}}^{\prime}=\hat{H}_{\mathrm{EBH}} / N^{2}$ (i.e., proportional to the energy per particle pair in the classical limit). Dropping the primes then gives the rescaled Hamiltonian expressed in the rescaled variables the same form as (1) with $Q_{1}=0$ and $Q_{3}=1$, but with $Q_{2}$ replaced by $Q_{2} / N$. Thus, to have a welldefined, finite classical limit, $Q_{2} / N$ must be of the same order as $Q_{4}$ and $Q_{5}$, i.e., $Q_{2} / N \sim Q_{4} \sim Q_{5} \sim 1$ [27]. With these rescalings, replacing operators with expectation values turns (1) into the classical Hamiltonian of Ref. [5]. The classical model is known to have exact one-site and symmetric two-site compactons under the parameter restrictions $Q_{2} / N=-4 Q_{5}$ and $Q_{2} / N=-2 Q_{5}$, respectively [5].

Quantum lattice compactons. Consider $N$ bosons in a one-dimensional periodic lattice (e.g., a ring) with $f$ sites. We then define a (hypothetical) $m$-site QLC as an eigenstate to Hamiltonian (1) with absolute certainty of finding all $N$ particles on $m$ consecutive sites. For a translationally invariant system all eigenstates must satisfy the Bloch theorem. Using the translation operator $\hat{T}$ and its eigenvalues $\tau_{k}$ (with $\tau_{k}^{f}=1$; $k$ integer, $0 \leqslant k \leqslant f-1$ ), a QLC with a given $\tau_{k}$ must therefore have the form

$$
\left|\Phi_{\mathrm{c}}\right\rangle=\sum_{\gamma} c_{\gamma} \frac{1}{\sqrt{M_{\gamma}}} \sum_{j=0}^{f-1} \tau_{k}^{j} \hat{T}^{j}\left|n_{1}^{(\gamma)}, \ldots, n_{m}^{(\gamma)}, 0, \ldots, 0\right\rangle .
$$

Here $\gamma$ denotes the different ways of placing $N$ particles at the $m$ first lattice sites, $n_{p}^{(\gamma)}$ denotes the number of particles at site $p$ for the $\gamma$ th basis state, and $M_{\gamma}$ its normalization factor [28]. States that are almost compactons will contain small contributions from additional states, denoted $\left|\psi_{\lambda}\right\rangle$, that are not located on $m$ sites. Using (2), we may express such eigenstates as

$$
|\Phi\rangle=\left|\Phi_{\mathrm{c}}\right\rangle+\sum_{\lambda} d_{\lambda}\left|\psi_{\lambda}\right\rangle
$$

where $d$ is used instead of $c$ for the probability amplitude of states not located on $m$ sites. For a true $m$-site QLC, $\sum_{\gamma}\left|c_{\gamma}\right|^{2}=1$. The quantity $\left|c_{m \text { site }}\right|^{2} \equiv \sum_{\gamma}\left|c_{\gamma}\right|^{2}$ is therefore a good measure of how compactonlike a state is, and will be called the ( $m$-site) compactness.

Since states with a high degree of localization (quantum breathers) for attractive nonlinearity generally appear among the lowest-energy eigenstates $[13,14]$, we expect to find them among the highest-energy states in the repulsive case. In particular, for the repulsive version of the classical model of Ref. [5], the highest-energy stationary state has, depending on parameter values, its main peak either on one or two sites (the transition corresponds to exchange of stability between one-site and two-site states [5]), and for special parameter values it compactifies into either a one-site or a two-site compacton [5]. Therefore, we should expect to see, in different regimes, quantum counterparts to one-site as well as two-site classical compactons in the highest-energy state of the repulsive (1) (equivalent to the ground state of the attractive model).

Let us stress that, in contrast to their classical counterparts but analogously to quantum breathers, the QLC (2) is translationally invariant, but compact in terms of correlations. Moreover, similarly to classical discrete compactons, the QLC (2) is compact in the space of Wannier functions defining the lattice of the EBH model, but generally not strictly compact in real (continuous) space due to the finite decay rate of Wannier functions [29].

One-site compacton. The one-site QLC is the simplest possible, since there is only one way to place all particles at one site, and thus Eq. (2) takes the form

$$
\left|\Phi_{\mathrm{c}}\right\rangle=\frac{1}{\sqrt{f}} \sum_{j=0}^{f-1} \tau_{k}^{j} \hat{T}^{j}|N, 0, \ldots, 0\rangle .
$$

To search for conditions to have exact QLC eigenstates, we rewrite the Hamiltonian (1) in the form $\hat{H}_{\mathrm{EBH}}=\sum_{p}\left(\hat{H}_{p}^{(0)}+\right.$ $\left.\hat{H}_{p}^{(1)}+\hat{H}_{p}^{(2)}\right)$, where

$$
\begin{aligned}
& \hat{H}_{p}^{(0)}=Q_{1} \hat{N}_{p}+Q_{3} \hat{N}_{p}^{2}+4 Q_{4} \hat{N}_{p} \hat{N}_{p+1}, \\
& \hat{H}_{p}^{(1)}=\left(\hat{a}_{p}^{\dagger} \hat{a}_{p+1}+\hat{a}_{p+1}^{\dagger} \hat{a}_{p}\right)\left[\frac{Q_{2}}{2}+2 Q_{5}\left(\hat{N}_{p}+\hat{N}_{p+1}-1\right)\right], \\
& \hat{H}_{p}^{(2)}=Q_{4}\left[\left(\hat{a}_{p+1}^{\dagger}\right)^{2}\left(\hat{a}_{p}\right)^{2}+\left(\hat{a}_{p}^{\dagger}\right)^{2}\left(\hat{a}_{p+1}\right)^{2}\right] .
\end{aligned}
$$

The superscript indicates how many particles the operator effectively tunnels. Obviously, when the parts of the Hamiltonian that tunnel one and two particles $\left[\hat{H}^{(1)}\right.$ and $\left.\hat{H}^{(2)}\right]$ act on the QLC state (4), states with particles spread over more than one site are generated. Thus, if (4) should be an eigenstate, then $\hat{H}^{(1)}\left|\Phi_{\mathrm{c}}\right\rangle=0$ and $\hat{H}^{(2)}\left|\Phi_{\mathrm{c}}\right\rangle=0$, yielding the parameter conditions

$$
\begin{aligned}
\frac{Q_{2}}{2}+2 Q_{5}(N-1) & =0, \\
Q_{4} & =0 .
\end{aligned}
$$

Taking the classical limit, condition (5a) corresponds to the condition to have classical one-site compactons [5], while there are no restrictions at all on $Q_{4}$ in the classical model. The disappearance of the last condition in the classical limit can be understood by considering the scaling of the different terms in $\hat{H}_{\mathrm{EBH}}$ with $N$ (for $Q_{2} / N \sim Q_{4} \sim Q_{5} \sim 1$ ) when acting on a one-site QLC: The terms associated with $Q_{2}$ and $Q_{5}$ scale as $N^{\frac{3}{2}}$, while those associated with $Q_{4}$ only scale as $N$. Thus, for a one-site QLC, $Q_{4}$ terms become negligible compared to the others for large $N$. 

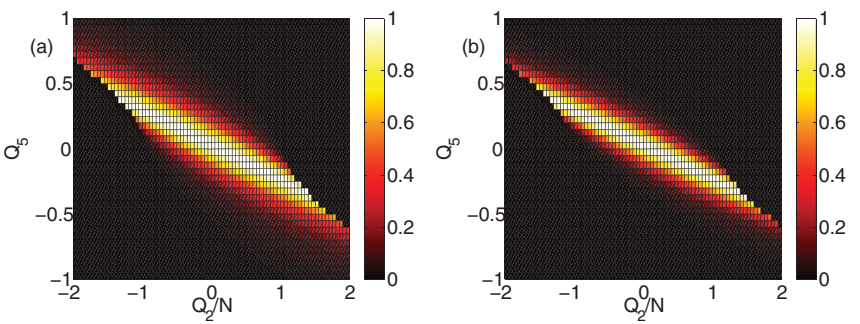

FIG. 1. (Color online) $\left|c_{1 \text { site }}\right|^{2}$ for the highest-energy eigenstate as a function of $Q_{2} / N$ and $Q_{5}$ with $Q_{4}=0$ for $f=4$ and (a) $N=16$ and (b) $N=26$ particles. $\tau_{k}=1$.

To illustrate the effects of deviations from conditions (5), we first show in Fig. 1 numerically obtained one-site compactness of the highest eigenstate when $Q_{4}=0$, for a small lattice with $f=4$ (close to compactness, increasing the number of sites affects the results only marginally). For $\left|Q_{5}\right| \lesssim 0.37$, a maximum with $\left|c_{1 \text { site }}\right|^{2}=1$, corresponding to condition (5a), is clearly seen. The one-site QLC is always an exact eigenstate when conditions (5) are fulfilled, but the location of the corresponding eigenvalue in the spectrum (highest, next highest, etc.) depends on the precise parameter values, so that when $\left|Q_{5}\right| \gtrsim 0.37$ it has moved to a lower eigenstate.

The effect of violating condition (5b) is illustrated in Fig. 2, showing the compactness of the highest eigenstate with condition (5a) fulfilled (i.e., $Q_{2}$ is varied along with $Q_{5}$ ). Comparing Figs. 1 and 2 evidences, just as the classical model indicates, that the compactness depends more on condition (5a) than (5b). The sharp transition into black regions in Fig. 2 shows where the QLC, or a compactonlike state with $\left|c_{1 \text { site }}\right|^{2}$ close to 1 , moves to a lower eigenvalue. As for the classical model [5], the location of this transition depends strongly on $Q_{4}$.

The fact that the exact one-site QLC (4) only consists of one (translationally invariant) basis state gives rise to some distinct features. Due to the orthogonality of eigenstates, no other (noncompact) eigenstate can contain this specific basis state. Therefore, there will be no mixing between the QLC and other states, causing sharp transitions between the QLC and other eigenstates rather than the normally observed smooth transitions as parameters are varied. Thus, while avoided crossings typically are observed between eigenvalues

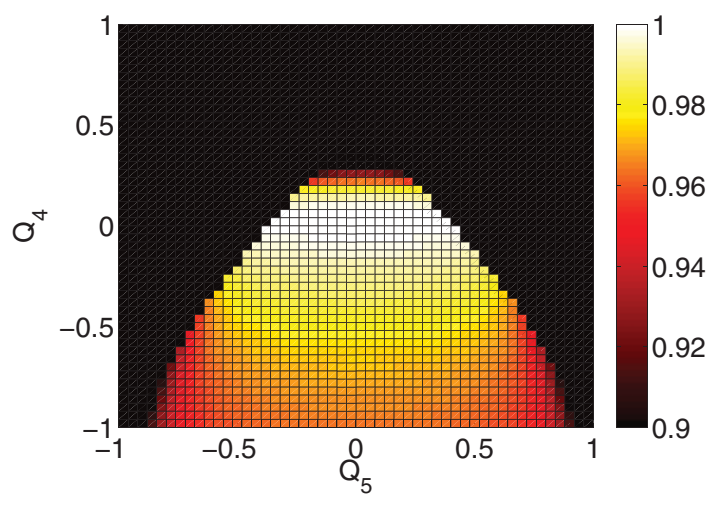

FIG. 2. (Color online) $\left|c_{1 \text { site }}\right|^{2}$ for the highest eigenstate when condition (5a) is fulfilled, for $N=16$ and $f=4$. Black regions have $\left|c_{1 \text { site }}\right|^{2}<0.9 . \tau_{k}=1$. corresponding to noncompact states, exact crossings appear for the QLC when conditions (5) are fulfilled [30].

Multisite compactons. To search for exact multisite QLCs is more complicated, since an $m$-site QLC (2) can be a superposition of different ways of placing all particles at $m$ consecutive sites.

Let us first attempt a similar strategy as for the onesite QLC, assuming that the Hamiltonian does not couple different basis states in the superposition (2) with each other. If parameters are tuned so that the coupling is turned off between the boundary sites [sites 1 and $m$ in (2)] and the first empty sites [sites $f$ and $(m+1)$ ] then, since the coupling is a function of the number of particles at the boundary sites, we need to have a boundary symmetric eigenstate, i.e., $n_{1}^{(\gamma)}=n_{m}^{(\gamma)}=n_{\text {boundary }}, \forall \gamma$. But the Hamiltonian will also try to tunnel particles inwards within the QLC [from site 1 to 2 and from site $m$ to $(m-1)]$, which will generate states that are not boundary symmetric. The only way to avoid this is to demand that $n_{2}=n_{m-1}=0$, implying that we have two isolated one-site QLCs at sites 1 and $m$, and not an $m$-site QLC. Repeating this argument shows that we cannot have a QLC with particles distributed on more than one consecutive site, in the absence of basis state coupling.

If one takes interactions between basis states into account, some exact multisite QLCs may indeed be found. However, by explicitly working through all possible tunneling processes [30], one finds that these QLCs must have very special forms. It can be proven that, as soon as $Q_{2} \neq 0$, a QLC (2) in a lattice that is more than twice as big as the QLC itself $(f>2 m)$ can contain at most three particles [30]. Thus, few-site QLCs with macroscopic occupation numbers (large $N$ ) can exist only for small lattices. Moreover, such QLCs generally are very asymmetric [e.g., for $m=2$ and $f=4$ a QLC containing only states of the form $|N-1,1,0,0\rangle$ exists under the same conditions (5) as the one-site QLC [30]]. Therefore, classical multisite compactons generally cannot correspond to exact QLCs.

To illustrate how classical multisite compactons may appear from noncompact quantum states, we show numerical results for $m=2$ and $f=4$. Figure 3 shows how the maximum two-site compactness for the highest-energy state increases with particle number $N$. The global maximum, obtained

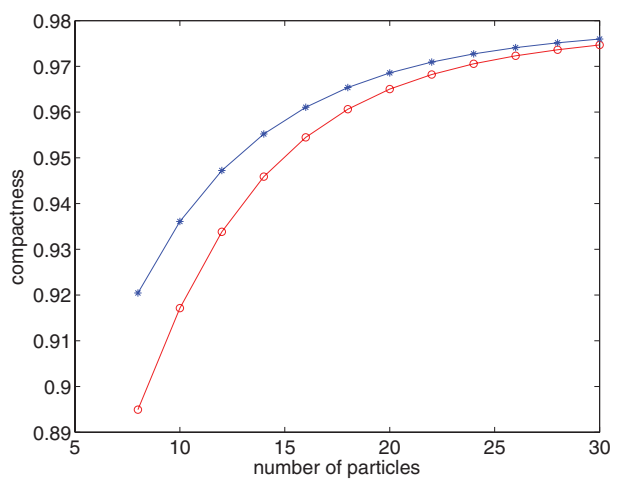

FIG. 3. (Color online) Maximum values of $\left|c_{2 \text { site }}\right|^{2}$ for the highestenergy state. Blue line (stars): Global max under independent parameter variations; red line (circles): $\max$ when $Q_{2}=-4 Q_{5}(N / 2-1)$. $f=4, \tau_{k}=1$. 

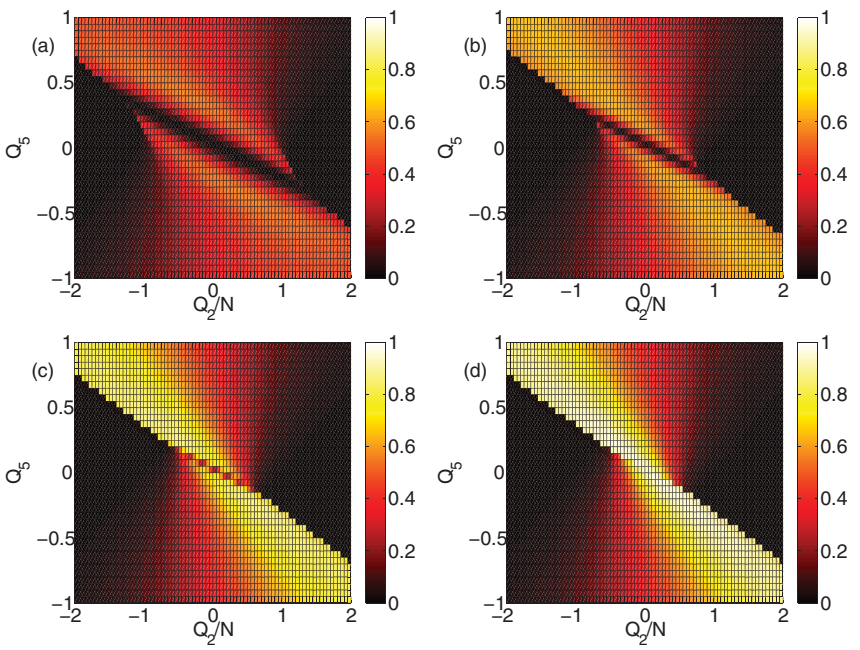

FIG. 4. (Color online) $\left|c_{2 \text { site }}\right|^{2}$ for the highest-energy state with $N=16$ and (a) $Q_{4}=0$; (b) $Q_{4}=0.2$; (c) $Q_{4}=0.3$; (d) $Q_{4}=0.4$. $f=4, \tau_{k}=1$.

from independent parameter variations of all $Q$ parameters, is compared to the maximum under the parameter restriction $Q_{2}=-4 Q_{5}(N / 2-1)$, corresponding to the classical twosite compacton condition [5]. In the classical limit, there is indeed a parameter regime where a two-site compacton maximizes the Hamiltonian [5], so we should expect both curves in Fig. 3 to approach unity as $N \rightarrow \infty$.

Figure 4 shows the two-site compactness of the highest eigenstate as a function of $Q_{2} / N$ and $Q_{5}$, for $N=16$ and different values of $Q_{4}$. Note that $\left|c_{2 \text { site }}\right|^{2}$ becomes close to unity only for $Q_{4}$ sufficiently large, reflecting the classical result that a threshold value of $Q_{4}$ is needed for a two-site localized state to become an energy maximizer [5]. The complementary nature of Figs. 4 and 1 thus gives a quantum mechanical illustration of the classical stability exchange between oneand two-site states. Note also the region with high compactness in Fig. 4 around the classical two-site compacton condition $Q_{5} \approx-Q_{2} / 2 N$.

A two-site compactonlike state (3) is mainly built up of twosite basis states, which we label as $|n\rangle=f^{-1 / 2} \sum_{j} \tau_{k}^{j} \hat{T}^{j} \mid N-$ $n, n, 0, \ldots, 0\rangle$. Figure 5 shows numerically obtained probability distributions for these basis states in compactonlike highest-
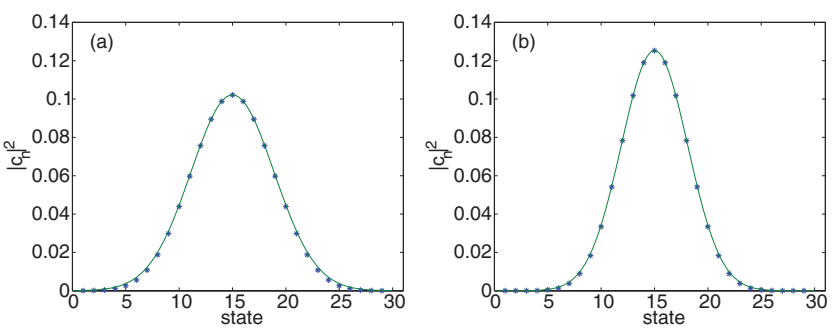

FIG. 5. (Color online) Probability distributions of two-site basis states (stars), matched with Gaussian functions (line), in the highestenergy eigenstates when $f=4, \tau_{k}=1, Q_{2}=-5, Q_{5}=0.08, N=$ 30 , and (a) $Q_{4}=0.4\left(\left|c_{2 \text { site }}\right|^{2}=0.9760\right)$; (b) $Q_{4}=0.6\left(\left|c_{2 \text { site }}\right|^{2}=\right.$ $0.9645)$.

energy states for two sets of parameter values with $N=30$, which are almost perfectly matched with Gaussian distributions $\exp \left[-\frac{(N / 2-n)^{2}}{2 \sigma^{2}}\right]$. Thus, symmetric basis states (equally many particles on both sites) are the most probable, and asymmetric ones get less probable with increasing asymmetry. Note, however, that the eigenstate with the highest compactness [Fig. 5(a)] is generally not associated with the narrowest Gaussian curve [Fig. 5(b)]. Consequently, one set of parameter values might give a state with higher probability to find all particles on two consecutive sites, while another set gives a state with higher probability to find the particles symmetrically distributed.

Conclusions. In conclusion, we have introduced the concept of QLC, and shown the correspondence between exact one-site QLC in an EBH model, and classical compactons of an extended DNLS model. Multisite compactons generically do not correspond to exact QLC, but were shown numerically to appear as superpositions dominated by states with all particles located on consecutive sites, with probability amplitudes following a Gaussian distribution. These results should pave the way for further theoretical investigations of QLC in other quantum lattice models and in higher dimensions, as well as stimulate an experimental search for signatures of strong bosonic quantum localization in lattice setups with significant pair correlation tunneling effects.

Acknowledgment. We thank J. C. Eilbeck, J. Karlsson, and A. Vega for discussions and assistance, and acknowledge support from the Swedish Research Council.
[1] S. Flach and A. V. Gorbach, Phys. Rep. 467, 1 (2008).

[2] A. Scott, Nonlinear Science (Oxford University Press, Oxford, 2003).

[3] P. Rosenau and J. M. Hyman, Phys. Rev. Lett. 70, 564 (1993); P. Rosenau, ibid. 73, 1737 (1994).

[4] P. G. Kevrekidis and V. V. Konotop, Phys. Rev. E 65, 066614 (2002); P. G. Kevrekidis, V. V. Konotop, A. R. Bishop, and S. Takeno, J. Phys. A: Math. Gen. 35, L641 (2002); P. G. Kevrekidis and V. V. Konotop, Math. Comput. Simul. 62, 79 (2003).

[5] M. Öster, M. Johansson, and A. Eriksson, Phys. Rev. E 67, 056606 (2003); M. Öster, Yu. B. Gaididei, M. Johansson, and
P. L. Christiansen, Physica D 198, 29 (2004); M. Öster and M. Johansson, Phys. Rev. E 71, 025601(R) (2005); Physica D 238, 88 (2009).

[6] F. Kh. Abdullaev, P. G. Kevrekidis, and M. Salerno, Phys. Rev. Lett. 105, 113901 (2010).

[7] D. Jaksch and P. Zoller, Ann. Phys. (NY) 315, 52 (2005).

[8] I. Bloch, J. Dalibard, and W. Zwerger, Rev. Mod. Phys. 80, 885 (2008).

[9] M. P. A. Fisher, P. B. Weichman, G. Grinstein, and D. S. Fisher, Phys. Rev. B 40, 546 (1989).

[10] D. Jaksch, C. Bruder, J. I. Cirac, C. W. Gardiner, and P. Zoller, Phys. Rev. Lett. 81, 3108 (1998). 
[11] M. Greiner, O. Mandel, T. Esslinger, T. W. Hänsch, and I. Bloch, Nature (London) 415, 39 (2002).

[12] V. M. Agranovich, in Spectroscopy and Excitation Dynamics of Condensed Molecular Systems, edited by V. M. Agranovich and R. M. Hochstrasser, Chap. 3 (North-Holland, Amsterdam, 1983), pp. 83-138.

[13] A. C. Scott and J. C. Eilbeck, Chem. Phys. Lett. 132, 23 (1986).

[14] R. A. Pinto and S. Flach, in Dynamical Tunneling: Theory and Experiment, edited by S. Keshavamurthy and P. Schlagheck, Chap. 14 (Taylor \& Francis, Boca Raton, FL, 2011).

[15] O. Morsch and M. Oberthaler, Rev. Mod. Phys. 78, 179 (2006).

[16] A. Trombettoni and A. Smerzi, Phys. Rev. Lett. 86, 2353 (2001).

[17] G. Mazzarella, S. M. Giampaolo, and F. Illuminati, Phys. Rev. A 73, 013625 (2006); G. Mazzarella, M. Moratti, L. Salasnich, and F. Toigo, J. Phys. B: At. Mol. Opt. Phys. 43, 065303 (2010).

[18] M. Eckholt and J. J. García-Ripoll, Phys. Rev. A 77, 063603 (2008); New. J. Phys. 11, 093028 (2009).

[19] J.-Q. Liang, J.-L. Liu, W.-D. Li, and Z.-J. Li, Phys. Rev. A 79, 033617 (2009); Y.-M. Wang and J.-Q. Liang, ibid. 81, 045601 (2010).

[20] X.-F. Zhou, Y.-S. Zhang, and G.-C. Guo, Phys. Rev. A 80, 013605 (2009).

[21] P.-I. Schneider, S. Grishkevich, and A. Saenz, Phys. Rev. A 80, 013404 (2009).

[22] S. Trotzky, P. Cheinet, S. Fölling, M. Feld, U. Schnorrberger, A. M. Rey, A. Polkovnikov, E. A. Demler, M. D. Lukin, and I. Bloch, Science 319, 295 (2008).
[23] A. Smerzi and A. Trombettoni, Chaos 13, 766 (2003); Phys. Rev. A 68, 023613 (2003).

[24] F. Kh. Abdullaev, Yu. V. Bludov, S. V. Dmitriev, P. G. Kevrekidis, and V. V. Konotop, Phys. Rev. E 77, 016604 (2008).

[25] As detailed, e.g., in Ref. [17], the tunneling coefficients decay asymptotically in the tight-binding limit as $Q_{2} \propto \epsilon, Q_{4} \propto \epsilon^{2}$, $Q_{5} \propto \epsilon^{3 / 2}$, where $\epsilon$ is an exponentially decreasing function of the lattice depth. Thus, for deep lattices $\left|Q_{4}\right|<\left|Q_{5}\right|<$ $\left|Q_{2}\right|$ generically, but for more shallow lattices it is possible to also have situations with $\left|Q_{4}\right|>\left|Q_{5}\right|$, while still being in a regime where the EBH model has good validity; cf. Refs. [21,22]

[26] The particular case $Q_{4}=Q_{5}=Q_{3} / 2$ also appears in a model for biphonons in the Fermi-Pasta-Ulam lattice; Z. Ivíc and G. P. Tsironis, Physica D 216, 200 (2006); X.-G. Hu and Y. Tang, Chin. Phys. B 17, 4268 (2008).

[27] See, e.g., Ref. [15] for a discussion how on-site interaction and single-particle tunneling energies may depend on particle number in experimental setups.

[28] Generally, $M_{\gamma}=f$, except for special cases when basis functions $|\gamma\rangle$ with $\hat{T}^{j}|\gamma\rangle=|\gamma\rangle$ for some $j, 0<j<f$, are included in $\left|\Phi_{\mathrm{c}}\right\rangle$.

[29] See, e.g., Ref. [8] for a discussion on the decay rate of Wannier functions in experimental setups.

[30] P. Jason, M.S. thesis, Linköping University (IFM), 2011, http://urn.kb.se/resolve?urn=urn:nbn:se:liu:diva-69500. 